\title{
The role of channel height and actuation method on particle manipulation in surface acoustic wave (SAW)-driven microfluidic devices
}

\author{
Citsabehsan Devendran ${ }^{1} \cdot$ David J. Collins $^{2} \cdot$ Adrian Neild $^{1}$ (I)
}

Received: 19 July 2021 / Accepted: 3 November 2021 / Published online: 13 January 2022

(c) The Author(s) 2022

\begin{abstract}
Surface acoustic wave (SAW) micromanipulation offers modularity, easy integration into microfluidic devices and a high degree of flexibility. A major challenge for acoustic manipulation, however, is the existence of a lower limit on the minimum particle size that can be manipulated. As particle size reduces, the drag force resulting from acoustic streaming dominates over acoustic radiation forces; reducing this threshold is key to manipulating smaller specimens. To address this, we investigate a novel excitation configuration based on diffractive-acoustic SAW (DASAW) actuation and demonstrate a reduction in the critical minimum particle size which can be manipulated. DASAW exploits the inherent diffractive effects arising from a limited transducer area in a microchannel, requiring only a travelling SAW (TSAW) to generate time-averaged pressure gradients. We show that these acoustic fields focus particles at the channel walls, and further compare this excitation mode with more typical standing SAW (SSAW) actuation. Compared to SSAW, DASAW reduces acoustic streaming effects whilst generating a comparable pressure field. The result of these factors is a critical particle size with DASAW $(1 \mu \mathrm{m})$ that is significantly smaller than that for SSAW actuation $(1.85 \mu \mathrm{m})$, for polystyrene particles and a given $\lambda_{\text {SAW }}=200 \mu \mathrm{m}$. We further find that streaming magnitude can be tuned in a DASAW system by changing the channel height, noting optimum channel heights for particle collection as a function of the fluid wavelength at which streaming velocities are minimised in both DASAW and SSAW devices.
\end{abstract}

Keywords Microfluidics $\cdot$ Acoustofluidics $\cdot$ Surface acoustic waves $\cdot$ Particle manipulation

\section{Introduction}

The small dimensions characteristic of microfluidic devices has enabled selective manipulation of similarly small objects such as cells and microparticles (Lee et al. 2017; Di Carlo 2009; Tayebi et al. 2020), including for tissue engineering (Choi et al. 2007; Andersson and van den Berg 2004; Khademhosseini et al. 2006; Novak et al. 2020; Bhatia and Ingber 2014), cell-cell interaction and signalling studies (Regehr et al. 2009; Faley et al. 2008; Zervantonakis et al. 2011), sample concentration and sorting (Ding et al. 2012;

Adrian Neild

adrian.neild@monash.edu

1 Department Mechanical and Aerospace Engineering, Monash University, Clayton 3800, Australia

2 Biomedical Engineering Department, University of Melbourne, Melbourne 3010, Australia
Gascoyne and Vykoukal 2002; Ahmed et al. 2018). This accurate and versatile manipulation of biological matter is essential for many lab-on-a-chip platforms, especially those designed for diagnostic purposes. A multitude of particle manipulation methods have been explored for manipulating specimens in microfluidic systems, covering both passive (Di Carlo 2009; Inglis et al. 2006; Davis et al. 2006) and active techniques (Baret et al. 2009; Inglis et al. 2004). The former relies on the configuration and design geometry of the microfluidic channels and sample inertia. Well-designed expansions, constrictions, weirs and pillars in strategic locations can alter the flow profiles to create favourable particle trajectories (Di Carlo 2009; Inglis et al. 2006; Davis et al. 2006). However, the dependence on these fixed features can lead to increased channel clogging and restrict the device's versatility, restricting it to specific tasks. In contrast, active methods are inherently more flexible, enabling on-demand actuation and allowing for a higher degree of selectivity that can be tuned for different applications. Consequently, a 
range of active manipulation methods have been established using magnetic (Xia et al. 2006; Wang et al. 2007), optical (MacDonald et al. 2003; Landenberger et al. 2012), electrical (Gascoyne and Vykoukal 2002; Shafiee et al. 2010) and acoustic forces (Collins et al. 2016; Ozcelik et al. 2018; Marzo et al. 2015).

Acoustofluidics, the application of acoustic fields in microfluidic devices, is especially promising due to its ease of integration on-chip and the ability to establish dexterous forces in a non-contact manner (Fakhfouri et al. 2018; Destgeer et al. 2015). Further, it is widely regarded as biocompatible when operated within the limits determined by the biological matter (Devendran et al. 2019). Hence, this manipulation approach has been extensively utilised to mix fluids (Zhang et al. 2019; Phan et al. 2015) as well as trap (Fakhfouri et al. 2016; Devendran et al. 2016), pattern and sort droplets (Sesen et al. 2017, 2015) and cells (Collins et al. 2015; Ahmed et al. 2016).

There are two main forces that are at play when manipulating suspended matter via acoustofluidic techniques, namely the acoustic radiation force and the acoustic streaming induced drag force (Muller et al. 2012). The former acts on the particles via direct interaction between the incident and scattered waves, whereas drag forces arise from the net steady flow of fluid caused by spatial variations in the sound field intensity. The relative strengths of these forces depends on the channel design, frequency of operation, fluid and particle properties, and the nature of the sound field (Muller et al. 2012; Settnes and Bruus 2012). In these systems, acoustic streaming dictates the minimum collectable particle size, with it having a far greater effect on disrupting the trajectories of particles towards a stable end location than Brownian motion (Tayebi et al. 2020).

To confine particles within specific locations or a band of streamlines, the acoustic radiation force should be dominant. As such, a limitation of the acoustic based manipulation technique arises due to the scaling of force with particle size, where $a$ is the particle radius. The acoustic radiation force, $F_{\mathrm{ARF}}$ scales in a standing wave system with $a^{3}$ (i.e. $\left.F_{\mathrm{ARF}, \mathrm{SW}} \propto a^{3}\right)$ (Gor'kov 1962) or in a travelling wave system to $a^{6}\left(F_{\mathrm{ARF}, \mathrm{TW}} \propto a^{6}\right)$ (Destgeer et al. 2013) for $a \ll \lambda$ (and non-linearly for $\mathcal{O}(a / \lambda) \sim 1$ ) (Ma et al. 2016), but only scales proportionally with drag force, $F_{\text {drag }}$ (i.e. $F_{\text {drag }} \propto a$ ). Therefore, while these forces decrease for particles smaller than the acoustic wavelength, the drag force does so at much a slower rate, hence, the dominant force with decreasing $a$, disrupting particle confinement. There is a great interest to develop acoustofluidic systems that minimises the relative strength of these negative streaming effects, and hence reduce the minimum critical particle size, $a_{\text {crit }}$ for which acoustic radiation force dominates.

Typically, at lower frequencies, ultrasonic fields are generated in microchannels using bulk acoustic wave (BAW) transducers that are operated such that resonance is established within a fluidic channel. For higher frequency acoustofluidic systems, $\mathcal{O}(10-600 \mathrm{MHz})$, surface acoustic waves (SAW) are typically used. This higher native frequency allows these devices to create acoustic fields whose wavelengths are on the order of individual particles and cells, where SAW-driven systems have gained widespread use given its efficient energy coupling into the fluid volume, simple integration, and its highly modular nature (in that additional functions, e.g. mixing or sensing, can be added by use of multiple IDT sets) (Zhang et al. 2019; Fu et al. 2010; $\mathrm{Xu}$ et al. 2018). In addition, the lack of need for fluid resonance offers a wider range of fields which can be excited. To achieve particle collection in confined regions, the most common implementation utilises two counter-propagating travelling SAWs (TSAW) excited by a pair of interdigital transducers (IDTs), which interfere constructively to produce a standing SAW (SSAW).

To reduce the $a_{\text {crit }}$ present in SSAW systems (for a given SAW wavelength, $\lambda_{\mathrm{SAW}}$ ) an unconventional configuration of SAW-driven acoustofluidic system is proposed. This method offers a relatively weak streaming field whilst ensuring strong particle confinement. In this configuration, the wave propagation direction is altered from being orthogonal to the channel length, to being aligned along it. Uniquely, whereas in SSAW the pressure field is generated due to interferences arising from counter-propagating wave fronts, the pressure field in our proposed case is due to interference arising from a spatially bounded TSAW, governed by the Huygens-Fresnel principle (Devendran et al. 2017). The wave fronts at any given point within the microchannel are the sum of spherical wave contributions emanating from an infinite number of discretized points on the transducer surface, resulting in a diffraction-driven pressure field. As such, we term this diffractive acoustic SAW (DASAW). DASAW principles have been demonstrated and explored in some of our recent work, principally from the perspective of predicting the spatial periodicity of the diffractive fringes that develop as a function of fluid/substrate properties, acoustic wavelength and the orientation of channel walls to the TSAW propagation direction (Devendran et al. 2017; Collins et al. 2018, 2019; O'Rorke et al. 2018; Devendran et al. 2020; Raymond et al. 2020). This present work, however, seeks to explore DASAW from the perspective of the sizedependent effects on particles, especially in comparison to those from more conventional SSAW-driven systems. We find that the DASAW configuration reduces $a_{\text {crit }}$ by reducing the relative magnitude of streaming velocities relative to SSAW-driven acoustic fields, whilst maintaining a similar acoustic radiation force; the $a_{\text {crit }}$ value for SSAW is $85 \%$ higher than that for DASAW. In addition, we numerically demonstrate that streaming velocities-and, therefore, the critical particle dimensions-are dependent on the channel 
height, finding discrete values at which the ratio of drag vs. acoustic forces is minimised (Muller et al. 2012), for both SSAW and DASAW.

\section{Operating principle}

The SAW-based acoustofluidic device consists of a $128^{\circ}$ Y-cut X-propagating lithium niobate $\left(\mathrm{LiNbO}_{3} ; \mathrm{LN}\right)$ piezoelectric crystal patterned with interdigital transducers (IDTs) bonded to the microchannel embedded within polydimethylsiloxane (PDMS). The SAW is typically generated via the application of an $\mathrm{A} / \mathrm{C}$ signal to the IDTs at a frequency corresponding to the desired wavelength. This propagating wave couples into the fluid at the Rayleigh angle, at the fluid-substrate interface, resulting in a pressure field within the fluid volume. The angle at which the wave propagates into the fluid is dictated by the discrepancy between the speed of sound in the fluid, $c_{\mathrm{f}}$ and substrate, $c_{\mathrm{s}}$ given by the Rayleigh angle, $\theta_{\mathrm{R}}=\sin ^{-1}\left(\mathrm{c}_{\mathrm{f}} / \mathrm{c}_{\mathrm{s}}\right)$.

However, as demonstrated recently by Devendran et al. (2017) and Collins et al. (2018), a complete description of the resultant sound field should consider the inherent diffractive effects imposed by the channel walls, orientation (Collins et al. 2019) and substrate (Fakhfouri et al. 2018). These diffractive effects have been shown to determine the radiation force periodicity (Fakhfouri et al. 2018, 2018). In this work, it is these diffractive effects which are exploited to form pressure fields in which small particles can be concentrated, in much smaller systems (sub wavelength width).

For conventionally driven SSAW systems (Fig. 1a(i)), diffractive effects have a secondary influence (in the $x$-propagation direction), rather the field is dominated by interference between counter-propagating waves, giving rise to the simulation boundary condition depicted in Fig. 1b-c(i) (see ESI Eq. S1), with both $x$ - and $z$-components (Devendran et al. 2016). As a result, a central anti-nodal region will be established in the channel. This configuration was chosen to provide a solid and clear comparison with its DASAW counterpart (please see ESI NOTE 3 for further details). In contrast, in the DASAW excitation configuration, the propagation $x$-direction is aligned with the channel axis and has minimal effects on the acoustic field in the channel crosssection, such that the boundary condition takes the form of a uniform harmonic displacement across the channel width at the LN/water interface (Fig. 1b-c(ii)). While diffractive effects due to finite IDT dimensions result in $y$-direction spatial displacement amplitude gradients in the transducer plane, these effects are minimal across channel widths on the order of a SAW wavelength, especially with increasing distance from the IDT (Fakhfouri et al. 2018). Furthermore, this is shown to have a minimal effect on the separation
Fig. 1 Acoustofluidic system for particle concentration illustrating a channel orientation with respect to the IDTs and SAW propagation direction (Note: Channel dimensions not to scale; $W=\lambda_{\text {SAW }} / 2$ ), b boundary conditions imposed on the fluid domain as used in the numerical simulations (see ESI Eqs. $\mathrm{S} 1$ and $\mathrm{S} 2$ for equations) and $\mathbf{c}$ the velocity boundary condition distribution (i.e. $\mathrm{v}_{\mathrm{BC}}{ }^{\mathrm{i}}{ }^{i \omega t}$ ) imposed on the bottom boundary (i.e. $z=0 \mu \mathrm{m}$ ) of the fluid domain (i.e. representing the LN substrate-fluid interface) for a (i) conventional SSAW and (ii) the DASAW system, respectively

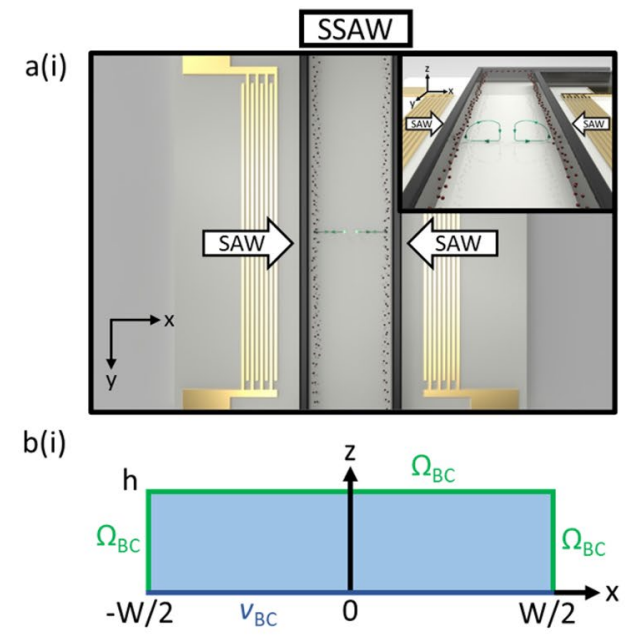

(ii)

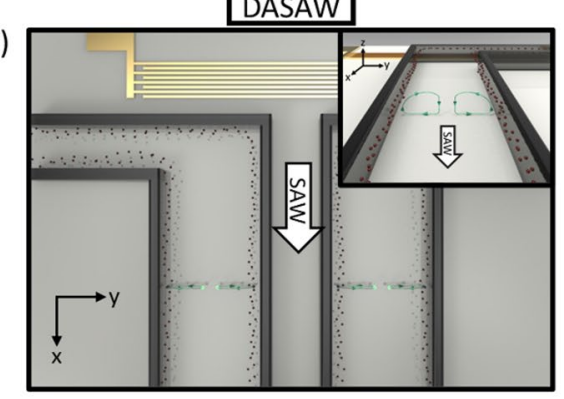

(ii)
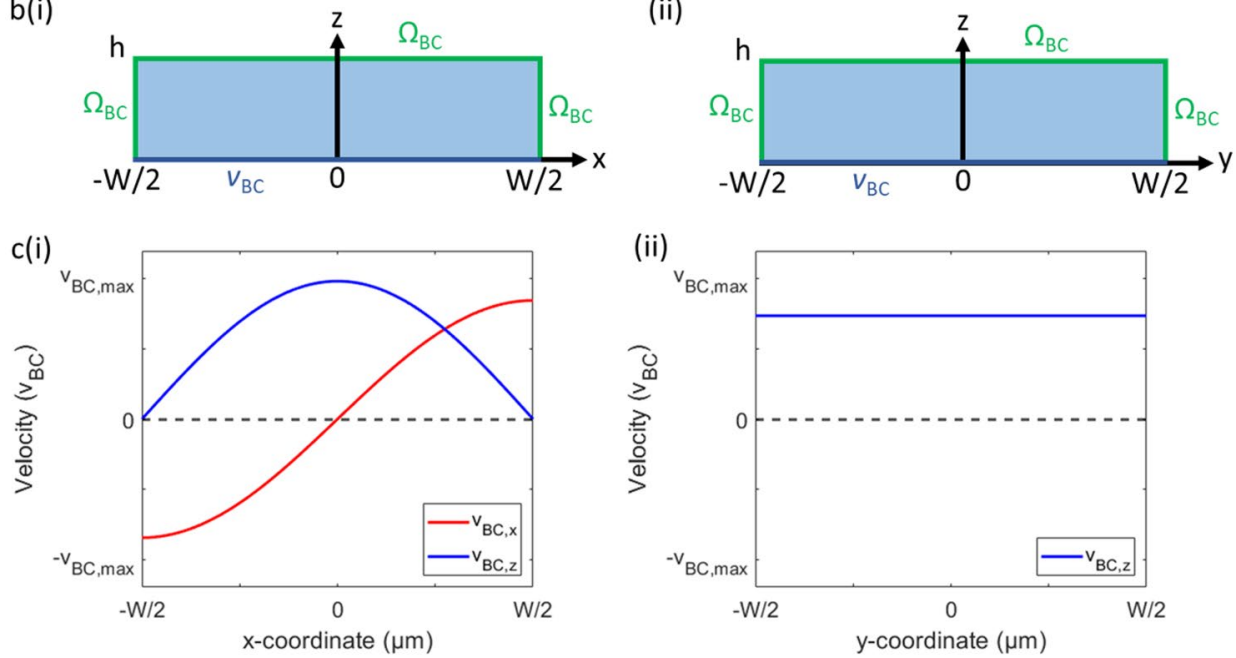
efficiency for the DASAW physical system (Devendran et al. 2020). Given these microchannels have a finite width, this equipotential wavefront couples into the fluid in a manner similar to a finite piston source. Whilst, a small difference in wave phase is expected (Vanneste and Bühler 2011) when modelling the system this way, ESI Fig. S1 demonstrates that accounting for the $x$-component of this propagation has little influence, where a 2D representation in which the oscillations are isotropically generated along the lower boundary results in an equivalent time-averaged pressure field to a 3D simulated case. One method of modelling the sound field is to consider this piston as producing both a plane wave, with edges waves (of inverse sign) from the periphery of the piston (Kramer et al. 1988). In this context, this is equivalent to a plane wave from the channel floor and a pair of cylindrical waves emanating from the lower corners of the channel. The result is a complex diffractive pressure field arising from a simple boundary condition. Whereas it is possible to set up an analytical expression for the pressure field according to this model (Collins et al. 2019), in this work we compute the pressure field numerically to account for channel reflections and acoustic concomitant acoustic streaming. In exploring the nature of streaming reduction through this excitation method, we also extend the study to account for changes in channel height.

\subsection{Time-averaged acoustic forces}

As a direct result of these coupled surface waves into the fluid, two distinct particle migration mechanisms arise. First, the acoustic radiation force stems from the pressure gradients in the acoustic field as it propagates and scatters upon interaction with a suspended matter within the fluid volume. If we consider a particle of radius a which is much smaller that the wavelength, $\lambda$ (i.e. $a \ll \lambda$ ), the radiation force takes the form of (Settnes and Bruus 2012):

$\mathbf{F}_{\mathrm{ARF}}=-\pi a^{3}\left[\frac{2 \kappa_{\mathrm{f}}}{3} \mathbb{R}\left[f_{1}^{*} \mathrm{P}_{1}^{*} \nabla \mathrm{P}_{1}\right]-\rho_{\mathrm{f}} \mathbb{R}\left[f_{2}^{*} v_{1}^{*} \nabla \boldsymbol{v}_{1}\right]\right]$,

where $\kappa_{\mathrm{f}}=1 /\left(\rho_{\mathrm{f}} c_{\mathrm{f}}^{2}\right)$ is the compressibility of the fluid, $\mathbb{R}[A]$ denotes the real part of the quantity $A$, the asterisk denote complex conjugates and factors $f_{1}$ and $f_{2}$ are given by

$f_{1}=1-\frac{\kappa_{p}}{\kappa_{\mathrm{f}}}$

and

$f_{2}=\frac{2(1-\gamma)\left(\rho_{p}-\rho_{\mathrm{f}}\right)}{2 \rho_{p}-\rho_{\mathrm{f}}(1-3 \gamma)}$,

where $\gamma=-\frac{3}{2}\left[1+i\left(1+\tilde{\delta}_{v}\right)\right] \tilde{\delta}_{v}$

$\tilde{\delta}_{v}=\frac{\delta_{v}}{a} ; \delta_{v}=\sqrt{\frac{2 \eta}{\omega \rho_{\mathrm{f}}}}$,

where $\kappa_{p}$ is the compressibility of the particle, $\delta_{v}$ is the viscous boundary layer and $\omega$ is the angular excitation frequency.

Second, acoustic streaming induced drag forces are responsible for entraining particles within the fluid motion due to the perturbations imposed by the ultrasonic excitation. This steady state fluid flow is due to the gradients in the first-order acoustic field as it propagates through a viscous fluid, resulting in a time-independent body force acting on the fluid (Nyborg 1953). Neglecting wall effects, the drag force on a spherical particle can be determined by

$\mathbf{F}_{\text {drag }}=6 \pi \eta a\left(\boldsymbol{v}_{2}-\boldsymbol{v}_{p}\right)$,

where $\boldsymbol{v}_{2}$ is the second-order time-independent streaming velocity and is given by

$\langle\mathbf{F}\rangle=\rho_{\mathrm{f}}\left\langle\left(\boldsymbol{v}_{1} \cdot \nabla\right) \boldsymbol{v}_{1}+\boldsymbol{v}_{1} \nabla \cdot \boldsymbol{v}_{1}\right\rangle$

$\langle\mathbf{F}\rangle=\nabla\left\langle P_{2}\right\rangle+\beta \eta \nabla\left(\nabla \cdot \boldsymbol{v}_{2}\right)+\eta \nabla^{2} \boldsymbol{v}_{2}$.

Therefore, $\boldsymbol{v}_{2}$ can be determined by calculating the first order velocity fields, $\boldsymbol{v}_{1}$, and subsequently solving the thermoviscous set of equations to obtain the Reynolds stress, $\mathbf{F}_{\text {Reynolds }}$ (i.e. $\mathbf{F}=-\mathbf{F}_{\text {Reynolds }}$ ). By determining $\mathbf{F}_{\text {Reynolds }}$, we are able to obtain $\boldsymbol{v}_{2}$ (Eq. $4 \mathrm{~b}$ ), which in turn will allow us to calculate the $\mathbf{F}_{\text {drag }}$ as shown in Eq. 3.

\section{Numerical model}

To understand and probe the underlying physics of DASAW, a numerical model has been developed. A fully comprehensive model would require a full three-dimensional approach that considers the electrostatics, elastic and hydrodynamic effects. Such a model, however, would be prohibitive from a computational perspective. We therefore propose a model that captures the essential features of this system, and consists of the two-dimensional fluid volume with appropriate boundary conditions as shown in Fig. 1b. We find that the omission of the $x$-component displacement excitation for the DASAW system plays a minimal role as the pressure distribution in that direction is relatively homogenous (ESI Fig. S1). The PDMS walls and ceiling are replaced with impedance boundary condition commensurate with PDMS $\left(c_{\text {PDMS }}=1076.5 \mathrm{~m} \mathrm{~s}^{-1} ; \rho_{\text {PDMS }}=1030 \mathrm{~kg} \mathrm{~m}^{-3}\right)$. This condition is justified here, as the typical PDMS wall thickness 
used experimentally (Collins et al. 2016; Barnkob et al. 2018; Devendran et al. 2016; Muller et al. 2012) is significantly thicker than that of the attenuation decay length (i.e. $\approx 580 \mu \mathrm{m}$ for a $f=19.97 \mathrm{MHz} ; \lambda_{\mathrm{SAW}}=200 \mu \mathrm{m}$ ) (Tsou et al. 2008). The bottom boundary $(z=0 \mu \mathrm{m})$ of the domain is represented by a distinct velocity boundary condition, $v_{\mathrm{BC}}$ as given in Fig. 1c for the two excitation modes, SSAW and DASAW, respectively. The $v_{\mathrm{BC}}$ for the conventional SSAW (Fig. 1c(i)) is given by the actuation profile discussed in Devendran et al. (2016). In contrast, the $v_{\mathrm{BC}}$ for the DASAW system (Fig. 1c(ii)) is given by an oscillating equipotential distribution. This $v_{\mathrm{BC}}$ represents the profile of the TSAW surface-bound wavefront in the far field (i.e. multiple $d^{2} / \lambda_{\text {SAW }}$ away from the source), which is of an equal magnitude across the channel width provided the channel width is smaller than that of the IDT aperture. This equipotential boundary condition is similar to that of BAW-driven systems, though BAW systems require reflecting boundary conditions to produce channel resonance, rather than the pseudo-absorbing boundaries provided by the PDMS at the channel walls and ceiling in Fig. 1b(ii). This absorption means that effects arising from reflections are reduced, and so diffraction effects become more prominent. To better compare the DASAW and SSAW actuation methods, the SSAW maximum velocity, $v_{\mathrm{BC} \text {,max }}$, is scaled by a factor of 0.68 , such that the maximum first-order time-averaged pressure, $\left\langle\left|\mathrm{P}_{1}\right|\right\rangle_{\max }$ is the same as for the DASAW system.

Using the stipulated boundary conditions, the fluid domain is solved for using the thermoviscous set of equations (thermoacoustic module in COMSOL Multiphysics) to accurately capture the resultant first-order acoustic and velocity fields. The first-order fields are then used to calculate the resultant body force (Eq. 4a). This body force is imposed throughout the domain, and the resultant streaming field is calculated using the Laminar Flow module in COMSOL Multiphysics. Consequentially, the particle trajectories are simulated using COMSOL Multiphysics' Particle Trajectory module by imposing the acoustic radiation forces (based on Eq. 1) and the drag forces (based on Eq. 3) for polystyrene particles of varying radius, $a$ to analyse the critical particle size, $a_{\text {crit }}$. This approach is similar to that of Muller et al. (2012) and Devendran et al. (2016). The corresponding channel height is consequentially varied to study the relative strengths of the resultant streaming fields, whilst maintaining the channel width to $\lambda_{\mathrm{SAW}} / 2$.

\section{Results and discussion}

\subsection{Pressure and streaming fields}

Solving for the thermoviscous set of equations as described in the Numerical Model section (Sect. 3), we obtain the first order pressure field, $\mathrm{P}_{1}$ for both systems for a given SAW wavelength $\left(\lambda_{\mathrm{SAW}}=200 \mu \mathrm{m}\right)$ and channel geometry ( $W=100 \mu \mathrm{m} ; h=50 \mu \mathrm{m})$ as shown in Fig. 2a. Here we observe a distinct distribution of the instantaneous pressure fields as the excitation (i.e. $v_{\mathrm{BC}}$ ) conditions are different (Fig. 1c). However, in both cases, pressure waves propagate upwards, towards the channel roof with minimal reflections, due to the PDMS impedance boundary condition (properties provided in Table S1). A low reflection coefficient at this interface is expected due to the small acoustic impedance mismatch between water and PDMS (Collins et al. 2016), and the high level of attenuation within the PDMS. However, a particle's migration is dependent on the timeaveraged field and not its instantaneous field. Accordingly, the former, $\left\langle\left|\mathrm{P}_{1}\right|\right\rangle$ is depicted in Fig. $2 \mathrm{~b}$ for both systems. Despite observable differences in $\left\langle\left|\mathrm{P}_{1}\right|\right\rangle$ between the SSAW (Fig. 2b(i)) and DASAW configurations (Fig. 2b(ii)), the core structure of a high pressure region in the central part of the channel with relatively low pressure regions at the channel periphery is observed in both cases. Setting the SSAW boundary conditions such that an acoustic antinode forms in the channel centre, as done here, therefore permits the most straightforward comparison between this actuation mode and DASAW.

Using Eq. 4a, we obtain the body force (Fig. 2c) based on the known first order fields. As shown in Fig. 2c (zoomed-in view of the bottom boundary; $z \approx 0 \mu \mathrm{m}$ ), we observe a significantly different body force distribution. For the SSAW configuration (Fig. 2c(i)), the body force ( $x$-direction) is mainly distributed relatively evenly around points a quarter of the width across the channel (i.e. $x= \pm \mathrm{W} / 4$ ). In contrast, the DASAW's body force distribution (Fig. 2c(ii)) has a second feature closer to the channel periphery, whilst retaining a similar but narrower distribution at the quarter width location (i.e. $x= \pm \mathrm{W} / 4$ ). The opposing nature (i.e. body force acting in the opposite direction of the centrally located body forces) of the feature at the channel periphery is the main reason a subdued streaming field is expected. The simulation shows a significantly weaker streaming field for the DASAW system (Fig. 2d(ii)) compared to that of the conventional system (Fig. 2d(i)), albeit for a similar flow profile (Fig. 2d).

We observe a significant reduction in streaming velocities via DASAW actuation compared to the SSAW system whilst maintaining a similar acoustic radiation field. Therefore, a reduced region that is streaming dominated (ESI Figs. S2 and S3), a reduced $a_{\text {crit }}$ is expected. Conducting particle trajectory analysis, for particles subjected to both acoustic radiation forces and streaming induced drag forces as shown in Fig. 3, a distinct difference in particle behaviour for a given size, $a$ is observed. For a particle of radius of $1 \mu \mathrm{m}$, the SSAW system (Fig. 3a) results in streaming dominated behaviour (rotational trajectories; see ESI Supplementary Video 1), whereas, the DASAW system (Fig. 3e) exhibits radiation force dominated 
Fig. 2 Numerical simulation results depicting the a first order pressure field, $\mathrm{P}_{1}$ (in $\mathrm{kPa}$ ), b the time-averaged absolute first order pressure, $\left\langle\left|\mathrm{P}_{1}\right|\right\rangle$ field (in $\mathrm{kPa}$ ), $\mathbf{c}$ the resultant body force (in $\mathrm{N} \mathrm{m}^{-1}$ ) distribution in the $x$-direction (arrows depict force in $x$ and $y$ direction) (Note: zoomed in view at the bottom boundary; $z \approx 0 \mu \mathrm{m}$ ) and $\mathbf{d}$ the resultant absolute streaming field, $\boldsymbol{v}_{2}$ (in $\mathrm{mm} \mathrm{s}^{-1}$ ) (arrows depict flow in the width direction (i.e. $x$ for SSAW; $y$ for DASAW) and height direction (i.e. $z$ )) for the (i) SSAW and (ii) DASAW system. All results are for a channel with a $W=100 \mu \mathrm{m}, h=50 \mu \mathrm{m}, \lambda_{\mathrm{SAW}}=$ $200 \mu \mathrm{m}$. The excitation amplitude was scaled to ensure the maximum $\left\langle\left|\mathrm{P}_{1}\right|\right\rangle$ is the same in both configurations for the sake of comparison

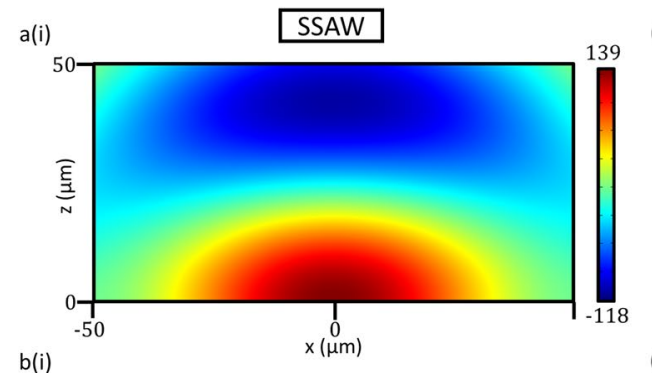

b(i)
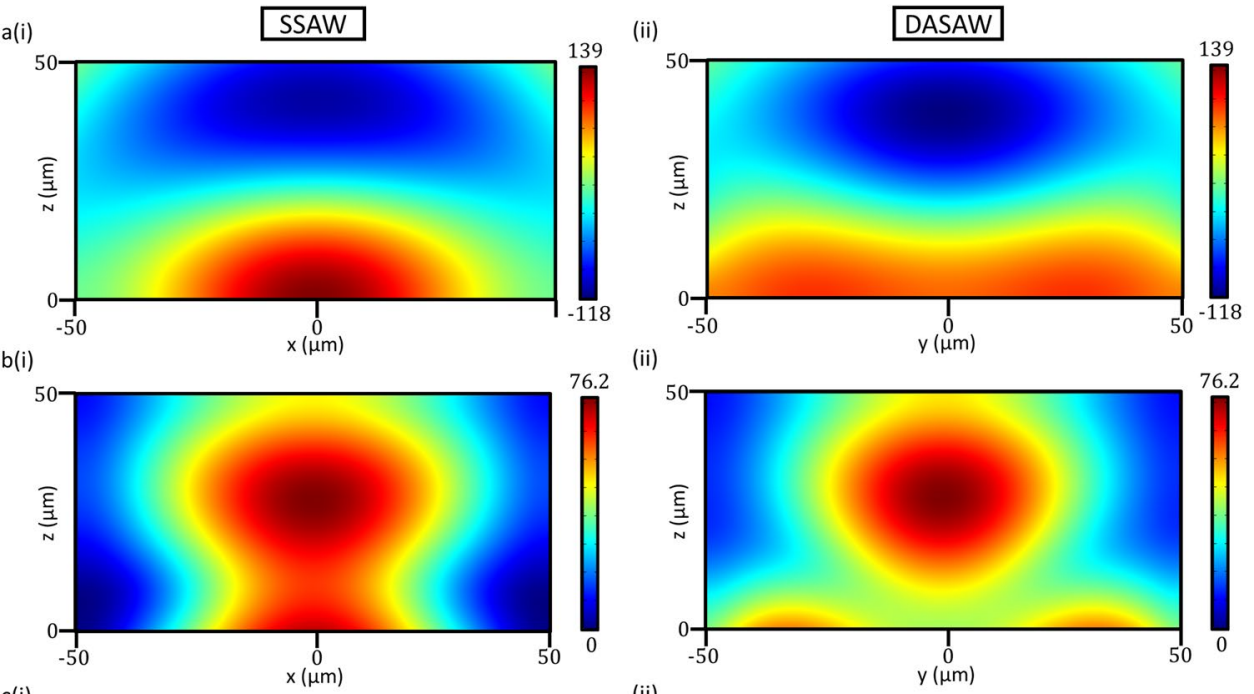

(ii)

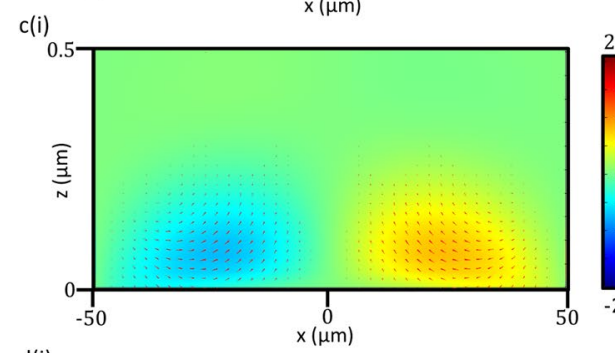

(ii)
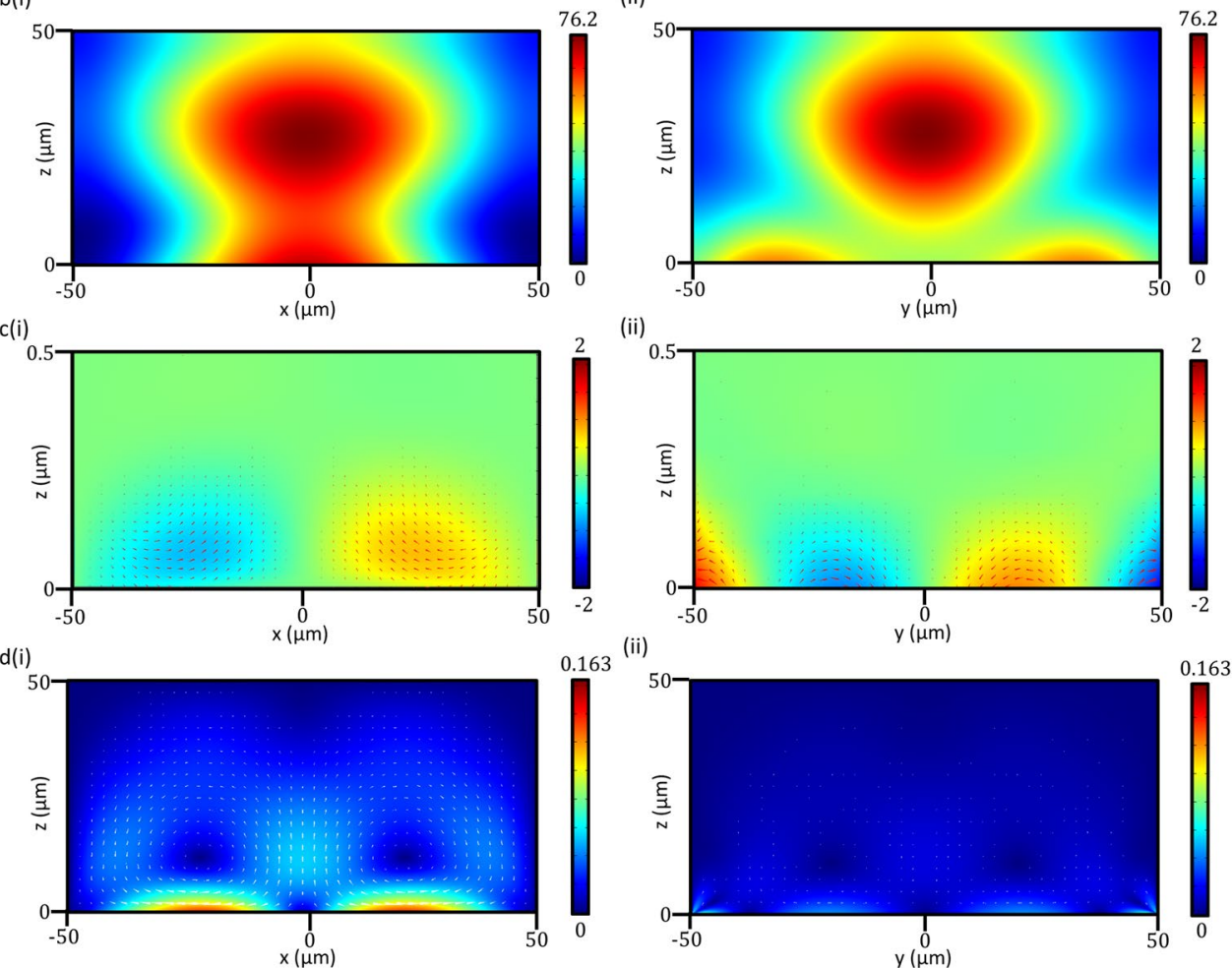

particle behaviour (see ESI Supplementary Video 2) (both with the same $\left\langle\left|\mathrm{P}_{1}\right|\right\rangle_{\max }, \lambda_{\mathrm{SAW}}=200 \mu \mathrm{m}$ ) and with distinct end locations (Fig. 3a(ii), e(ii)). Consequently, the $a_{\text {crit }}$ for the SSAW system is $1.85 \mu \mathrm{m}$ (see Fig. $3 \mathrm{~b}$ ), whereas the $a_{\text {crit }}$ for the DASAW system is significantly lower at $1 \mu \mathrm{m}$ (see Fig. 3e), a reduction of $46 \%$. The critical particle size will vary based on the particle properties (density, speed of sound, diameter) and channel geometry as well. For the sake of a valid comparison, we kept these parameters constant. Additionally, while smaller particles comprised of materials with higher acoustic impedance will result in a lower critical particle diameter, the acoustic properties of polystyrene used here are more representative of cells than metal particles used in other work (Sazan et al. 2019).

\subsection{Height dependence: comparison between the SSAW and DASAW}

In BAW-based acoustofluidic systems with rectangular cross-sections (Muller et al. 2012), acoustic waves propagate parallel to the two surfaces that do not contribute to resonance. This grazing of the surface means that, in the dimension between these surfaces, there is a steep decline in particle velocity from the large uniform amplitude in the bulk of the fluid. This decline, which takes place over the viscous penetration depth, $\delta_{v}$ creates very strong first order fluid velocity gradients in the vicinity of the channel walls; hence, the acoustic streaming fields are driven by this effect, known as boundary layer streaming (Schlichting streaming). In contrast, in a SAW system, propagation is primarily in the direction at which the energy is coupled from the substrate, the Rayleigh angle, and so inclined to all internal channel surfaces. Therefore, streaming has been shown to be independent of boundary layer streaming effects (Devendran et al. 2016; Nama et al. 2015), but rather driven by the first-order acoustic field gradients in the fluid that give rise to the body force (i.e. Reynolds stress) distributions. The lack of boundary layer-driven streaming for SAW systems, coupled with the asymmetric nature (in the height, $z$-direction) of excitation, results in the streaming strength 

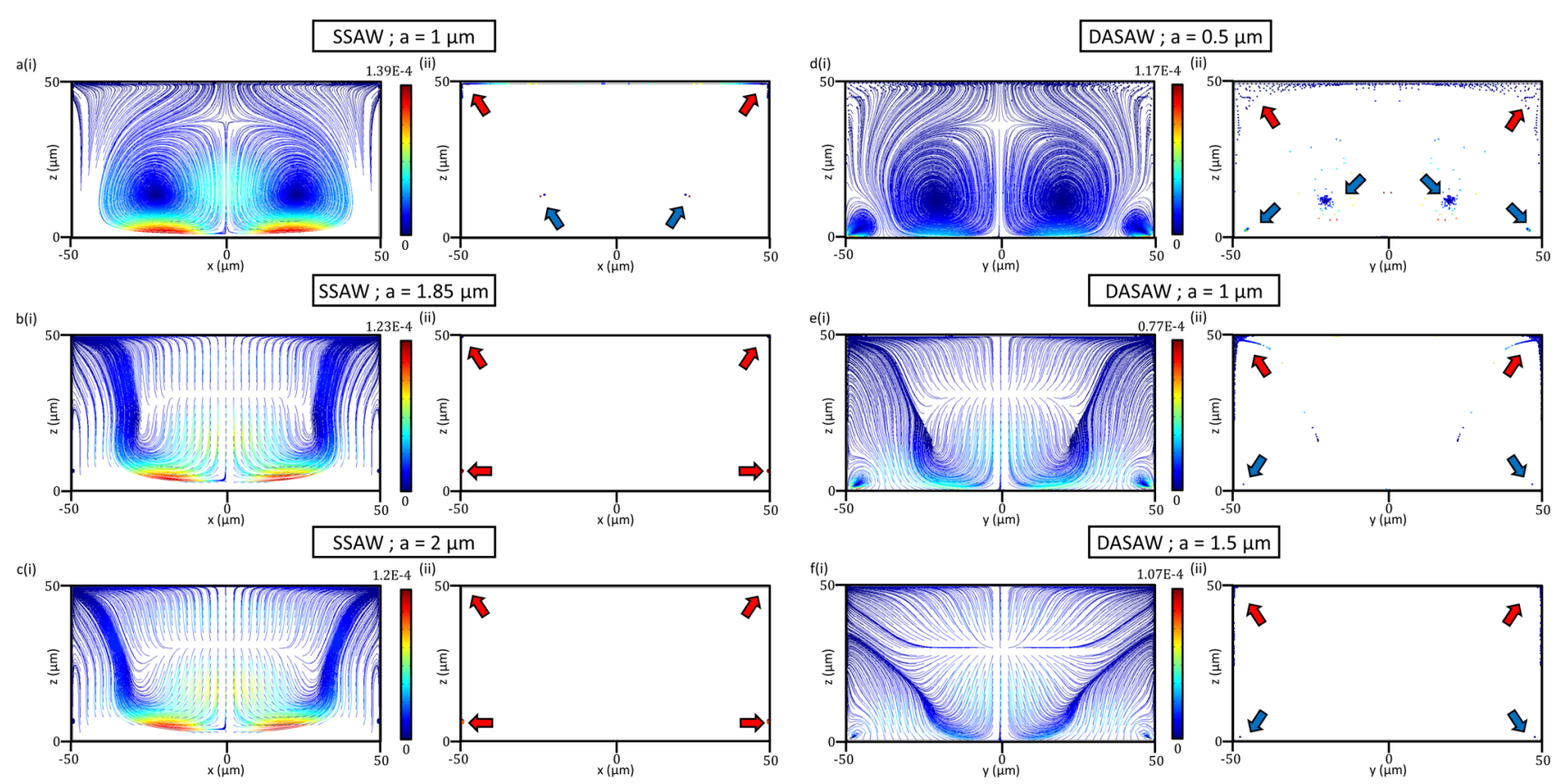

Fig. 3 Particle trajectory (i) and end location (ii) after $20 \mathrm{~s}$ for particles of radius a $1 \mu \mathrm{m}, \mathbf{b} 1.85 \mu \mathrm{m}\left(a_{\text {crit }}\right)$, c $2 \mu \mathrm{m}$ for the SSAW excitation and d $0.5 \mu \mathrm{m}$, e $1 \mu \mathrm{m}\left(a_{\text {crit }}\right)$, f $1.5 \mu \mathrm{m}$ for the DASAW excitation. Red arrows dictate end particle location dominated by radiation forces, and blue arrows show stable recirculation location dominated

being height dependent. Second, the body force distribution between the SSAW platform and DASAW platform differs as shown in Fig. 2c. The body force distribution in the SSAW platform (Fig. 2c(i)) is due to the surface velocity profile at the substrate-fluid interface (i.e. $z=0 \mu \mathrm{m}$ ) and the resultant first-order velocity gradients (in the $x$-direction). The $z$ and $x$-components of the $v_{\mathrm{BC}}$ are symmetric about the channel centre (i.e. $x=0 \mu \mathrm{m}$ ), thus generating an inward (towards the channel centre) body force. In contrast, the DASAW platform (Fig. 2c(ii)) is driven solely by an equipotential $z$-component oscillation. The body force distribution is due to the discontinuity associated with the finite width of the microchannel, rather than being dominated by a $y$-component velocity akin to the SSAW system.

Figure 4 summarises the effect of channel height on streaming. Figure 4a(i) shows the absolute streaming velocity profile along a vertical line (as depicted by the black dashed line in Fig. 4a(ii)) for the conventional configuration. Channel heights, $h$, ranging from $h=5 \mu \mathrm{m}$ to $h=100 \mu \mathrm{m}\left(h_{\text {stepsize }}=5 \mu \mathrm{m}\right)$ are inspected for a $\lambda_{\text {SAW }}=200$ $\mu \mathrm{m}, W=100 \mu \mathrm{m}$ SSAW configured system. For the sake of clarity, the velocity profiles begin at a height $z=0.2 \mu \mathrm{m}$ and end at $z=h$. This lower limit is imposed to demonstrate the velocity distribution along the vertical line clearly, without clutter close to $z \approx 0 \mu \mathrm{m}$ where all the velocities converge to $\boldsymbol{v}_{2}=0 \mathrm{~mm} \mathrm{~s}^{-1}$ (no-slip boundary). Figure $4 \mathrm{a}$ (iii) (Note

by streaming induced drag forces. All results are for a channel with a $W=100 \mu \mathrm{m}, h=50 \mu \mathrm{m}, \lambda_{\text {SAW }}=200 \mu \mathrm{m}$. See ESI Supplementary Video 1 and 2 for animations depicting resultant particle trajectories. See ESI Fig. S5 for comparisons of the same particle sizes

the scale differs on the $z$-axis by a factor of 100 and in the $x$-axis by a factor of 2 compared to a(ii)) clearly demonstrates the sharp gradient in velocities approaching $0 \mathrm{~mm}$ $\mathrm{s}^{-1}$ at this boundary $(z=0 \mu \mathrm{m})$ as expected at the fluid-substrate interface.

The corresponding maximum streaming velocity for each channel height is plotted in Fig. $4 \mathrm{~b}$ for both the SSAW and DASAW configurations $\left(\lambda_{\mathrm{SAW}}=200 \mu \mathrm{m}\right)$. The location of the maximum streaming is consistently close to the channel bottom $(z \leq 0.2 \mu \mathrm{m})$, as expected due to this being the location of the largest gradient in the first order acoustic velocity fields. The SSAW configuration results in a larger absolute streaming velocity across all channel heights studied. This is consistent with the streaming simulations in Fig. 2c. A similar trend with a reduced absolute velocity is observed for the $2^{\text {nd }}$ local maxima. The increasing trend (Fig. $4 \mathrm{~b}$; the $x$-axis is normalised to the fluid wavelength, $\lambda_{\mathrm{f}}\left(\lambda_{\mathrm{f}} \approx 74.86\right.$ $\mu \mathrm{m} ; \lambda_{\mathrm{SAW}}=200 \mu \mathrm{m}$ ) for the sake of visual clarity and fair comparison) of streaming velocities for $\mathrm{h} \leq \frac{1}{3} \lambda_{\mathrm{f}}(\mathrm{h} \leq 25 \mu \mathrm{m}$ for $\lambda_{\text {SAW }}=200 \mu \mathrm{m}$ ) is due to the influence of the channel ceiling (i.e. no-slip boundary condition). This diminishes as the channel height increases, causing a lower resistance to fluid motion for the larger channel heights. However, upon reaching a channel height where this trend peaks $\left(\mathrm{h} \approx \frac{1}{3} \lambda_{\mathrm{f}}\right)$, the relationship inverts (as shown for $\frac{1}{3} \lambda_{\mathrm{f}} \leq \mathrm{h} \leq \frac{2}{3} \lambda_{\mathrm{f}}$ ). This is potentially due to the increase in the amount of fluid that 
Fig. 4 The a streaming velocity, $\boldsymbol{v}_{2}$ for (i) different channel heights (normalised by $\lambda_{\mathrm{f}}$ ) along the vertical line $x=21.76 \mu \mathrm{m}$ (indicated in black (ii); intersects minimum streaming location) and (iii) the zoomed in view (Note: different size scale) of the streaming velocity (in $\mathrm{mm} \mathrm{s}^{-1}$ ) distribution at the bottom boundary $\left(\lambda_{\text {SAW }}=200 \mu \mathrm{m}\right.$; $W=100 \mu \mathrm{m}$ for the SSAW system). Line plots comparing the b maximum streaming velocity (based on $\mathbf{a}(\mathrm{i})$ ) and $\mathbf{c}$ the vortex centre height as determined by the minimum absolute streaming velocity in $\mathbf{a}(\mathrm{i})$ as a function of channel height, $h$ for the conventional and proposed system $\left(\lambda_{\text {SAW }}=200 \mu \mathrm{m} ; W=100 \mu \mathrm{m}\right)$
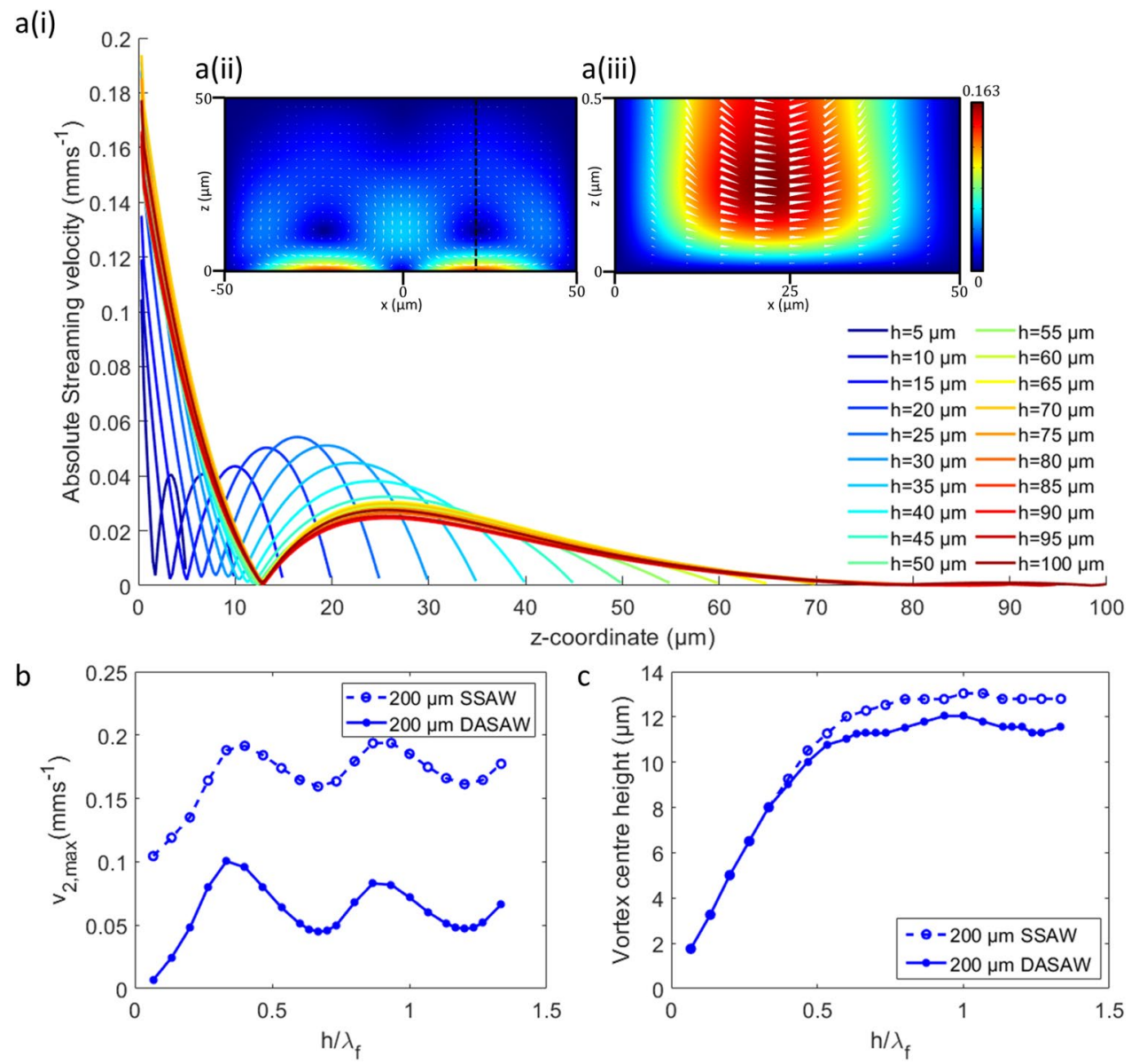

needs to be circulated. The relationship between streaming velocity and height subsequently oscillates with a regular periodicity as the channel height is further increased (Fig. 4b), with a weaker secondary counter-flow present for channel heights above $\mathrm{h}=\frac{2}{3} \lambda_{\mathrm{f}}$ (i.e. $50 \mu \mathrm{m}$ ), as well as the first-order acoustic velocity field. This periodicity is due to the inherent periodicity of the acoustic velocity, $v_{1}$ in the $z$-direction, and will be discussed in more detail in Sect. 4.4.

Second, we probe the location of the vortex centre as a function of channel height. As shown in Fig. 4c, an increasing trend is observed for increasing channel heights, $h$ before it begins to plateau out for heights above $50 \mu \mathrm{m}$ (i.e. $h \geq 50 \mu \mathrm{m}$ ). This is attributed to the channel ceiling's influence. First, an asymmetric velocity profile is expected due to the nature of a bottom-driven system. Second, as the channel height increases, the centre of vortex approaches its stable location (given the inherent asymmetry, this is not at $\frac{\mathrm{h}}{2}$ ). With increasing channel height, the vortex centre height increases to a stable value. A weaker secondary counter-flow is present for heights above $h=\frac{2}{3} \lambda_{\mathrm{f}}$ (i.e. $50 \mu \mathrm{m} ; \lambda_{\text {SAW }}=200 \mu \mathrm{m}$ ). A small discrepancy in centre of vortex location for these heights is observed when comparing the SSAW and DASAW configurations.

\subsection{Height dependence: effect of SAW wavelength}

Further investigation into the streaming velocities as a function of SAW wavelength for a given channel height is shown in Fig. 5. The resultant streaming fields for the DASAW configuration $\left(\lambda_{\text {SAW }}=200 \mu \mathrm{m}\right)$ with three distinct heights of $h=5 \mu \mathrm{m}$ (Fig. $5 \mathrm{a}(\mathrm{i})$ ), $h=50 \mu \mathrm{m}$ (Fig. 5a(ii)) and $h=100 \mu \mathrm{m}$ (Fig. 5a(iii)) are shown along with their corresponding probed velocity profile lines (Fig. 5a; white dashed lines). Here we observe similar trends in streaming velocities, as described in Sect. 4.2, for channel heights of 5 $\mu \mathrm{m}$ to $50 \mu \mathrm{m}$ (i.e. $\frac{1}{15} \lambda_{\mathrm{f}} \leq h \leq \frac{2}{3} \lambda_{\mathrm{f}}$ ). Again, this is due to the influence imposed by viscous drag with the channel ceiling. However, a further increase in channel height to $100 \mu \mathrm{m}$ as in Fig. 5a(iii), does not result in a significant increase in the streaming velocities, but a significantly weaker secondary counter-vortex develops in the upper section of the fluid domain.

Similar analysis is carried out as in Fig. 4b but for varying $\lambda_{\text {SAW }}$ as shown in Fig. $5 b$ (see ESI Fig. S4 for the raw data). A similar trend, to that described in Sect. 4.2, is observed for all $\lambda_{\text {SAW }}$ considered. Interestingly, with a smaller wavelength (and thus higher frequency) (Dentry et al. 2014; 
Fig. 5 a Surface plots depicting the resultant streaming velocity, $\boldsymbol{v}_{2}$ (in $\mathrm{mm} \mathrm{s}^{-1}$ ) distribution for the three distinct heights, $h$ (i) $10 \mu \mathrm{m}$, (ii) $50 \mu \mathrm{m}$ and (iii) $100 \mu \mathrm{m}$ for the $\lambda_{\mathrm{SAW}}=200$ $\mu \mathrm{m}$ proposed system. Channel width, $W=100 \mu \mathrm{m}$ (i.e. $\lambda_{\text {SAW }}$ /2). Line plots comparing the b maximum streaming velocity (based on commensurate data as in Fig. 4a(i)) as a function of normalised channel height (normalised to the corresponding wavelength in the fluid) and $\mathbf{c}$ the vortex centre height as determined by the minimum absolute streaming velocity in Fig. 4a(i) as a function of channel height, $h$ for the conventional and proposed system $\left(\lambda_{\text {SAW }}=\right.$ as indicated in legend; $\left.W=\lambda_{\mathrm{SAW}} / 2\right)$ a(i)

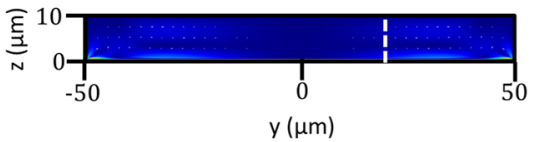

a(ii)

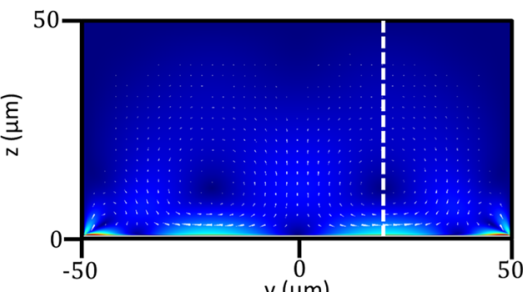

b

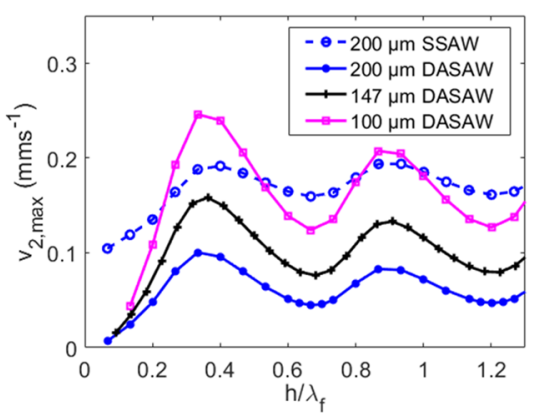

a(iii)

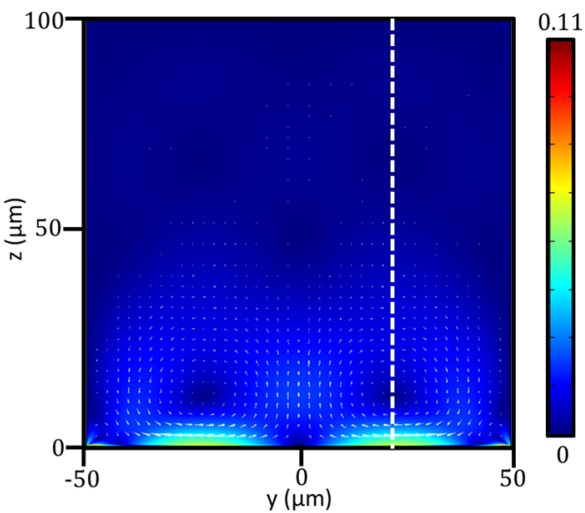

C

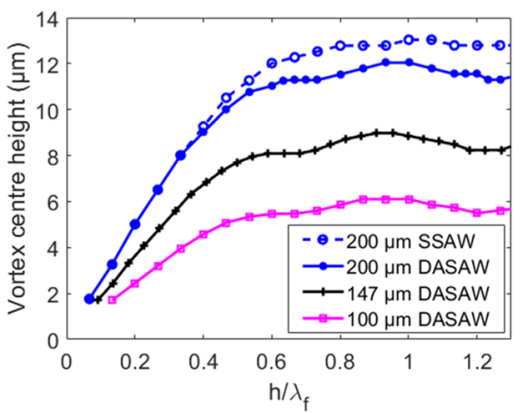

Collins et al. 2016) of $\lambda_{\text {SAW }}=147 \mu \mathrm{m}$ in a DASAW system, the resultant streaming velocities are consistently lower than that of the $\lambda_{\mathrm{SAW}}=200 \mu \mathrm{m}$ SSAW excited system. However, the $\lambda_{\text {SAW }}=200 \mu \mathrm{m} \mathrm{SSAW} \mathrm{results} \mathrm{in} \mathrm{comparable} \mathrm{streaming}$ velocities to a $\lambda_{\text {SAW }}=100 \mu \mathrm{m}$ DASAW system (Fig. $5 \mathrm{~b}$ ). This observation is key to an increased performance of particle concentration as an increased radiation force is expected with an increased frequency. This is achieved whilst maintaining similar or even reduced streaming velocities, driving $a_{\text {crit }}$ lower. However, a more pronounced undulation (Fig. 5b) in the maximum streaming velocity occurs as a function of channel height, as the frequency of operation is increased (i.e. $\lambda_{\text {SAW }}$ is decreased). This is potentially due to the larger gradients in the first-order acoustic velocity fields, as expected with a decreasing fluid wavelength (Dentry et al. 2014). The centre of vortex as shown in Fig. 5c has a consistent trend for all $\lambda_{\text {SAW }}$. As $\lambda_{\text {SAW }}$ is decreased, the vortex centre height decreases as well. This is once again attributed to the reduced fluid wavelength, thus, a reduced penetration depth.

\subsection{Optimum channel height}

Here, we seek to identify the optimum channel heights whereby streaming velocities, and thus $a_{\text {crit }}$ minimised. We examine this for the DASAW configuration as it has a lower $a_{\text {crit }}$ than SSAW. The oscillatory trend in maximum streaming velocity as observed in Figs. $4 \mathrm{~b}$ and $5 \mathrm{~b}$ is probed to elucidate on the oscillatory behaviour observed as a function of channel height. We note 3 distinct features that are noteworthy for a scaled system (with respect to $\lambda_{\mathrm{SAW}}$ ) analogous to that depicted in Fig. 2. These features are (1) the existence of a channel height dependence on the resultant streaming strength (Figs. $4 \mathrm{~b}, 5 \mathrm{~b}$ ), (2) the presence of a spatially split dominance (i.e. concurrent observation of streaming and radiation dominated behaviour in the lower and upper channel sections, respectively) beyond a certain channel height (i.e. $h \geq \frac{2}{3} \lambda_{\mathrm{f}}$ ) and (3) a complex particle behaviour close to the channel periphery as a result of being subjected to the resultant forces (i.e. particle confinement at the channel periphery despite a streaming dominated behaviour) for channel heights, $h \leq \frac{1}{3} \lambda_{\mathrm{f}}$. These features will now be discussed in more detail. From here on, the system is analysed for $\lambda_{\text {SAW }}=100 \mu \mathrm{m}(W=50 \mu \mathrm{m})$; however, the underlying relationships hold consistent for all $\lambda_{\mathrm{SAW}}$, provided the width and height are scaled with $\lambda_{\text {SAW }}$ and $\lambda_{\mathrm{f}}$, respectively.

\subsubsection{Feature 1: the relationship between height and streaming field}

The streaming velocities are as a direct result of the gradients present in the first-order acoustic velocity, $\boldsymbol{v}_{1}$ fields as given by Eq. 4 a. First, we note that the streaming field strength is a function of channel height. The $v_{1}$ field for a $h=12.5 \mu \mathrm{m}$, as shown in Fig. 6a(i), results in the first (and global) maxima in streaming velocities, $v_{2}$ (Fig. $\left.6 \mathrm{~b}\right)\left(\lambda_{\mathrm{SAW}}=100 \mu \mathrm{m}\right.$; $W=50 \mu \mathrm{m})$. This is due to the relatively lower opposing body force (horizontally outwards from $y=0 \mu \mathrm{m}$ ) due to the minimal $v_{1}$ gradient present at the channel ceiling (i.e. $\mathrm{z} \approx h$ ). A $v_{1}$ local minima is present close to this height (Fig. 6a(i)), 
which corresponds to the location of the pressure maxima, $P_{\text {max }, 1}$ due to diffractive effects (see ESI Fig. S6). The first $P_{\max , 1}$ in the $z$-direction at the channel centre (i.e. $y=0 \mu \mathrm{m}$ ), thus, the height that the maximum streaming occurs at first, $h_{\text {max }, 1}$ for a channel width, $W=50 \mu$ m (i.e. $\lambda_{\text {SAW }} / 2$ ) coincides with

$$
\begin{gathered}
\mathrm{h}_{\mathrm{max}, 1}=\sqrt{\left[\left(\left(\frac{3}{4}\right)\left(\frac{3_{\mathrm{c}_{\mathrm{f}}}}{4_{\mathrm{c}_{\mathrm{SAW}}}}\right)\right)^{2}-\left(\frac{1}{4}\right)^{2}\right]} \lambda_{\mathrm{SAW}} \\
\text { for } \mathrm{W}=\frac{\lambda_{\mathrm{SAW}}}{2}
\end{gathered}
$$

This equation is derived (see ESI Fig S4) based on the interference of apparent edge with vertically propagating wave fronts, discussed in detail elsewhere (Collins et al. 2019), and is a consequence of the finite width of the transducer region. Importantly, Eq. 5 holds for channel widths of
$W=\lambda_{\mathrm{SAW}} / 2$. For widths above this criterion, the pressure and velocity fields are significantly more complex and thus, has an irregular trend (Collins et al. 2018). As the objective of the proposed DASAW excitation configuration is to concentrate particles, this is done by confining them at the channel periphery (Fig. 3e, f) akin to that of a SSAW excitation (Fig. 3b, c). To achieve similar pressure field distributions (Fig. 2b) the channel width is constrained to $\lambda_{\text {SAW }}$ /2. Importantly, the subsequent $h_{\min , 1}$ corresponds to double the height of $h_{\text {max }, 1}$ as shown in Fig. 6a(ii) as a significant counter-gradient (i.e. opposing the dominant direction) is present at the channel ceiling (i.e. $z \approx h$ ), thus, reducing the streaming strength driven by the large acoustic gradients present at the channel floor (i.e. $\mathrm{z} \approx 0$ ). This channel height corresponds to the ideal height if the critical particle size dominated by acoustic streaming induced drag forces are to be minimised. As, the channel heights increase further, the effects driven by diffraction are reduced due to attenuation and is dominated by the fluid wavelength, $\lambda_{\mathrm{f}}$ in the vertical
Fig. 6 Surface plots of a first order velocity fields, $v_{1}$ for channel heights of (i) $12.5 \mu \mathrm{m}$ (first local maxima), (ii) $25 \mu \mathrm{m}$ (first local minima), (iii) 32.5 $\mu \mathrm{m}$ (second local maxima) and (iv) $45 \mu \mathrm{m}$ (second local minima) for of a $\lambda_{\mathrm{SAW}}=100$ $\mu \mathrm{m}, W=50 \mu \mathrm{m}$. All surface plots are restricted to the same first-order velocity range, in $\mathrm{mm}$ $\mathrm{s}^{-1}$ for the sake of comparison. Determination of $\mathbf{b} \Delta_{\mathrm{h}} \mathrm{v}_{2 \text { in }}$ based on the channel height difference between streaming velocity local minima as illustrated in black dash lines (insets denote the corresponding local maxima (green arrow) and minima location (purple arrow)) for $\lambda_{\mathrm{SAW}}$ $=100 \mu \mathrm{m}$ and $\mathbf{c}$ comparison between the predicted (based on Eq. 6) and numerical predictions for a given $\lambda_{\mathrm{SAW}}$ (for $\lambda_{\mathrm{SAW}}$ $=100 \mu \mathrm{m}, 147 \mu \mathrm{m}$ and $200 \mu \mathrm{m}$ )
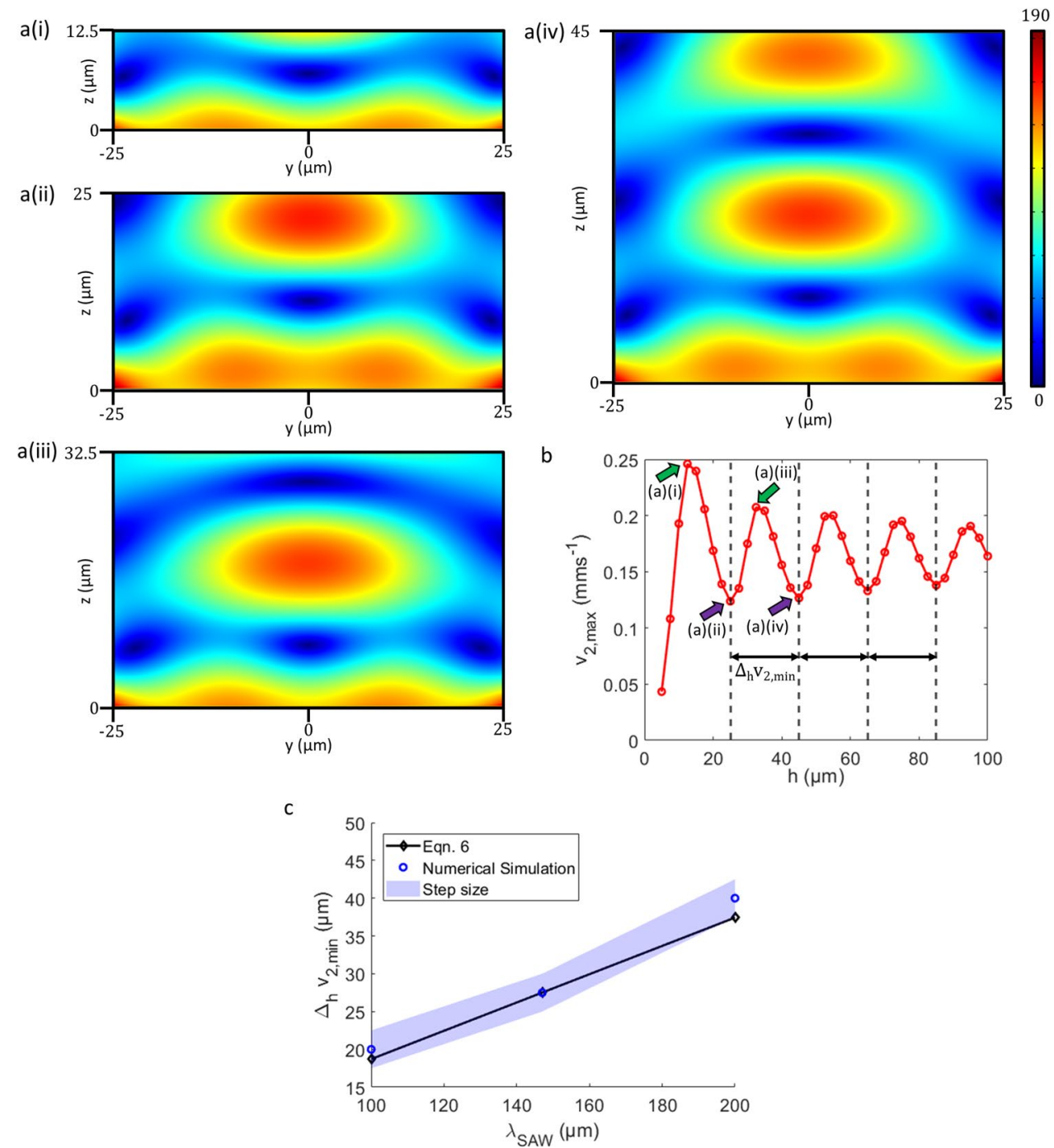
direction (i.e. $z$-direction) (see Fig. 6a(iii-iv) for the next local maxima and minima, respectively). Subsequently, the corresponding $h_{\text {min, } \mathrm{n}}$, where $n$ is an element of integer is given by multiples of the half-fluid wavelength, $\frac{(n-1) \lambda_{\mathrm{f}}}{2}$, as follows:

$\mathrm{h}_{\min , \mathrm{n}}==_{\mathrm{h}_{\min , 1}}+\frac{(n-1) \lambda_{\mathrm{f}}}{2}$.

As depicted in Fig. 6c, the predicted channel height corresponding to the $\Delta_{\mathrm{h}} \mathrm{v}_{2, \text { min }}$ (dictated by the distance between the dashed black lines in Fig. 6b) via simulations is denoted by the blue circle. The theoretical values as calculated based on Eq. 6, are given by the black diamond marker and black lines. Both, the predicted theoretical value and the numerical simulation results for the $\Delta_{\mathrm{h}_{2, \text { min }}}$, are well within the step size (i.e. $h_{\text {stepsize }}=2.5 \mu \mathrm{m}$ ) of the channel height increments in the numerical simulation. To better test this hypothesis, an arbitrarily chosen $\frac{1}{2} \lambda_{\mathrm{f}}=27.5 \mu \mathrm{m}$ is used and to specify a corresponding $\lambda_{\text {SAW }}=147 \mu \mathrm{m}$ (Fig. 6c). As postulated, both the theoretical and simulation prediction of $\Delta_{\mathrm{h}} \mathrm{v}_{2, \text { min }}$, align well at the specified $\lambda_{\mathrm{f}}$. Therefore, to minimise the effects of streaming, the channel height should be designed such that it conforms to these local minima.

\subsubsection{Feature 2: spatial force dominance variance}

The second phenomena that should be noted is although the periodic nature of $\mathbf{v}_{2, \max }$ as a function of channel height extends far beyond a channel height of $25 \mu \mathrm{m}$ (for $\lambda_{\mathrm{f}}=100 \mu$ $\mathrm{m}$ ), we observe that the dominant force varies spatially across the channel, whereby the bottom section of the fluid volume experiences streaming dominated behaviour and a radiation dominated behaviour above these heights (i.e. $z \geq$ $25 \mu \mathrm{m})$ (Fig. 7b, c(iii-iv)). This is due to the existence of a secondary streaming vortex which is significantly weaker (as shown in Fig. 5a(iii)) than the main streaming vortex close the channel floor which only extends to a fluid height of $z \approx$ $30-35 \mu \mathrm{m}$ (assuming $h$ is larger).

\subsubsection{Feature 3: complex behaviour at the channel periphery}

Further testing the hypothesis that reducing the relative streaming strength would result in a reduction of $a_{\text {crit }}$, we analyse the particle trajectory subjected to their corresponding acoustic radiation forces and the streaming induced drag forces. We note more complex particle behaviour as a result of being subjected to these forces. Although, the particle trajectory for a $0.7 \mu \mathrm{m}$ radius particle is clearly streaming dominated in the bulk of the fluid for a $h=12.5 \mu \mathrm{m}$ (Fig. 7b(i)) as opposed to a radiation dominated trajectory for a $h=25 \mu \mathrm{m}$ (Fig. 7b(ii)), consistent with the predictions made in Fig. 6b, the end location (at $4 \mathrm{~s}$ ) of the particles are similar (i.e. at the channel periphery) as shown in Fig. 7c(i-ii). This is due to the associated increase in acoustic radiation forces at play close to the channel periphery for the lower channel heights. As shown in Fig. 7a, the location of the maxima in the timeaveraged pressure field distribution corresponding to the first streaming local maxima channel height $(h=12.5 \mu \mathrm{m}$; Fig. 7a(i)) is close to the channel floor (i.e. $z \approx 0 \mu \mathrm{m}$ ), whereas, for a $h=25 \mu \mathrm{m}$ (Fig. 7a(ii)) the maxima is located in the top half of the channel (i.e. $z>h / 2$ ). This inherent distribution has significant influence on the resultant particle trajectory as shown in Fig. 7b. We demonstrate the resultant particle trajectory of the spatially split dominance phenomenon (Feature 2) in Fig. 7(a-c)(iii-iv) for channel heights of $32.5 \mu \mathrm{m}\left(2^{\text {nd }}\right.$ local maxima) and $50 \mu \mathrm{m}\left(2^{\text {nd }}\right.$ local minima). Figure $7(\mathrm{a}-\mathrm{c})($ iii) clearly depicts streaming dominated behaviour in the bottom half of the channel, whilst exhibiting radiation force dominated behaviour in the top section. A similar behaviour is observed for the $50 \mu \mathrm{m}$ height channel (Fig. 7(a-c)(iv)), although relatively weaker streaming behaviour should be noted in Fig. 7b(iv).

Whilst, the reduction of $a_{\text {crit }}$ via the optimisation of channel height and operating in a DASAW configuration does alter the particle trajectory, it does not solely dictate the particle's end location. Although it is pertinent to understand the underlying physics at play regarding streaming fields and approaches to minimise it by means of altering the channel height, we have shown particle trajectories depends on spatially dominated effects as well (Feature 2 and 3; either by pressure field distributions or presence of weaker secondary vortices). Therefore, as the goal of the SSAW and DASAW configurations examined is to confine particles to the channel periphery for concentration purposes (Devendran et al. 2020), design sensitivity with respect to channel height should be considered along with particle end (collection) locations, whilst maintaining an effectively reduced $a_{\text {crit }}$. This would allow for microchannel designs with high-throughput systems, benefiting commercial and clinical translation.

\section{Conclusion}

DASAW is a novel approach exploiting inherent diffractive effects to excite acoustofluidic platforms as an alternative to SSAW configurations for particle manipulation. The DASAW approach reduces the critical particle size, $a_{\text {crit }}$, by reducing the inherent streaming effects, whilst maintaining a similar acoustic radiation field. Further, as the DASAW platform relies on diffractive effects, timeaveraged fields required for particle confinement can be established using TSAW, requiring only a single set of IDTs, further simplifying the control system. In this work, 


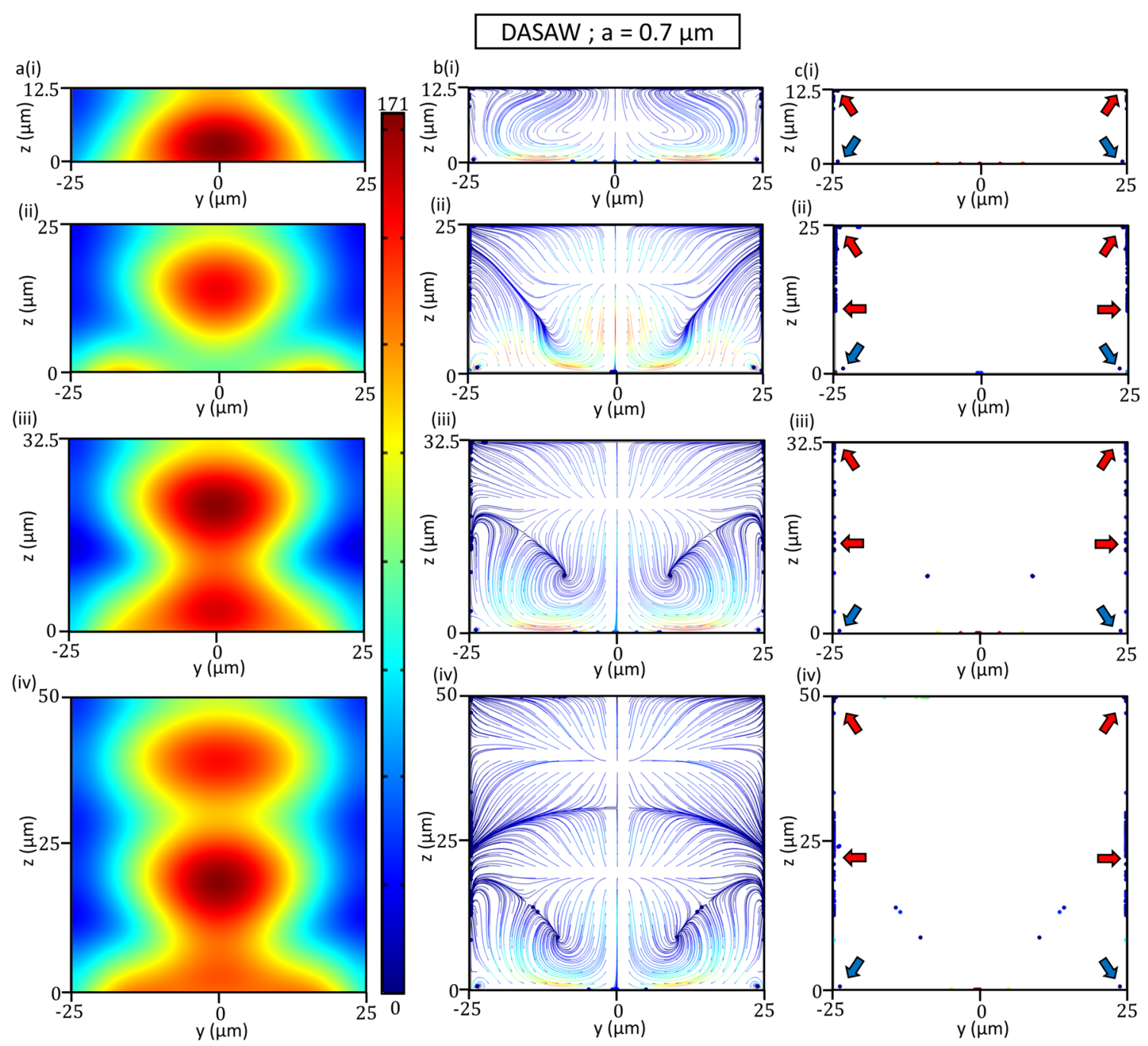

Fig. 7 Surface plots of a time-averaged first order pressure field, $\left\langle\left|\mathrm{P}_{1}\right|\right\rangle$ (in $\mathrm{kPa}$; scale kept consistent for comparison), b the trajectories of $0.7 \mu \mathrm{m}$ radius particles and $\mathbf{c}$ the end location after $4 \mathrm{~s}$ (Red arrows dictate end particle location dominated by radiation forces and blue arrows show stable re-circulation location dominated by stream- ing-induced drag forces) for the DASAW system with corresponding channel heights of (i) $12.5 \mu \mathrm{m}$ (first local maxima), (ii) $25 \mu \mathrm{m}$ (first local minima) (iii) $32.5 \mu \mathrm{m}$ (second local maxima) and (iv) $50 \mu \mathrm{m}$ (second local minima). $\lambda_{\text {SAW }}=100 \mu \mathrm{m}, W=50 \mu \mathrm{m}$ we compare and analyse these two distinct configurations numerically to reveal a $a_{\text {crit }}$ value for SSAW which is $85 \%$ higher than that of its DASAW counterpart $\left(\lambda_{\text {SAW }}\right.$ of 200 $\mu \mathrm{m})$, a significant improvement. We further demonstrate a channel height dependence on the streaming field, for a range of $\lambda_{\mathrm{SAW}}$, aiding the design process of these platforms. We define a relationship with $\frac{1}{2} \lambda_{\mathrm{f}}$ that predicts the corresponding channel height corresponding to a streaming strength local minima/maxima, and present a model that predicts channel heights at which the influence of acoustic streaming can be minimised. To decrease the $a_{\text {crit }}$, the corresponding local minima should be selected, minimising the disruptive effects of streaming induced drag forces.
Supplementary Information The online version contains supplementary material available at https://doi.org/10.1007/s10404-021-02509-9.

Acknowledgements This research was undertaken with the assistance of resources from the National Computational Infrastructure (NCI), which is supported by the Australian Government.

Funding Open Access funding enabled and organized by CAUL and its Member Institutions.

Open Access This article is licensed under a Creative Commons Attribution 4.0 International License, which permits use, sharing, adaptation, distribution and reproduction in any medium or format, as long as you give appropriate credit to the original author(s) and the source, provide a link to the Creative Commons licence, and indicate if changes were made. The images or other third party material in this article are 
included in the article's Creative Commons licence, unless indicated otherwise in a credit line to the material. If material is not included in the article's Creative Commons licence and your intended use is not permitted by statutory regulation or exceeds the permitted use, you will need to obtain permission directly from the copyright holder. To view a copy of this licence, visit http://creativecommons.org/licenses/by/4.0/.

\section{References}

Ahmed H, Destgeer G, Park J, Jung JH, Sung HJ (2018) Vertical hydrodynamic focusing and continuous acoustofluidic separation of particles via upward migration. Adv Sci 5(2)

Ahmed D, Ozcelik A, Bojanala N, Nama N, Upadhyay A, Chen Y, Hanna-Rose W, Huang TJ (2016) Rotational manipulation of single cells and organisms using acoustic waves. Nat Commun

Andersson H, van den Berg A (2004) Microfabrication and microfluidics for tissue engineering: state of the art and future opportunities. Lab Chip 4(2):98-103

Baret JC, Miller OJ, Taly V, Ryckelynck M, El-Harrak A, Frenz L, Rick C, Samuels ML, Hutchison JB, Agresti JJ, Link DR, Weitz DA, Griffiths AD (2009) Fluorescence-activated droplet sorting (FADS): efficient microfluidic cell sorting based on enzymatic activity. Lab Chip 9(13):1850-1858

Barnkob R, Nama N, Ren L, Huang TJ, Costanzo F, Kähler CJ (2018) Acoustically driven fluid and particle motion in confined and leaky systems. Phys Rev Appl 9(1):014027

Bhatia SN, Ingber DE (2014) Microfluidic organs-on-chips

Choi NW, Cabodi M, Held B, Gleghorn JP, Bonassar LJ, Stroock AD (2007) Microfluidic scaffolds for tissue engineering. Nat Mater 6:908-915

Collins DJ, O'Rorke R, Devendran C, Ma Z, Han J, Neild A, Ai Y (2018) Self-aligned acoustofluidic particle focusing and patterning in microfluidic channels from channel-based acoustic waveguides. Phys Rev Lett 120(7)

Collins DJ, Morahan B, Garcia-Bustos J, Doerig C, Plebanski M, Neild A (2015) Two-dimensional single-cell patterning with one cell per well driven by surface acoustic waves. Nat Commun 6:8686

Collins DJ, Ma Z, Ai Y (2016) Highly localized acoustic streaming and size-selective submicrometer particle concentration using high frequency microscale focused acoustic fields. Anal Chem 88(10):5513-5522

Collins DJ, Neild A, Ai Y (2016) Highly focused high-frequency travelling surface acoustic waves (saw) for rapid single-particle sorting. Lab Chip 16:471-479

Collins DJ, Devendran C, Ma Z, Ng JW, Neild A, Ai Y (2016) Acoustic tweezers via sub-time-of-flight regime surface acoustic waves. Sci Adv 2(7):e1600089

Collins DJ, O'Rorke R, Neild A, Han J, Ai Y (2019) Acoustic fields and microfluidic patterning around embedded micro-structures subject to surface acoustic waves. Soft Matter 15(43):8691-8705

Davis JA, Inglis DW, Morton KJ, Lawrence DA, Huang LR, Chou SY, Sturm JC, Austin RH (2006) Deterministic hydrodynamics: taking blood apart. Proc Natl Acad Sci USA 103(40):14779-84

Dentry MB, Yeo LY, Friend JR (2014) Frequency effects on the scale and behavior of acoustic streaming. Phys Rev E Stat Nonlinear Soft Matter Phys 89(1):013203

Destgeer G, Lee KH, Jung JH, Alazzam A, Sung HJ (2013) Continuous separation of particles in a PDMS microfluidic channel via travelling surface acoustic waves (TSAW). Lab Chip 13(21):4210-4216

Destgeer G, Ha BH, Park J, Jung JH, Alazzam A, Sung HJ (2015) Microchannel anechoic corner for size-selective separation and medium exchange via traveling surface acoustic waves. Anal Chem 87(9):4627-4632
Devendran C, Albrecht T, Brenker J, Alan T, Neild A (2016) The importance of travelling wave components in standing surface acoustic wave (SSAW) systems. Lab Chip 16(19):3756-3766

Devendran C, Gunasekara N, Collins D, Neild A (2016) Batch process particle separation using surface acoustic waves (SAW): integration of travelling and standing SAW. RSC Adv 6(7):5856-5864

Devendran C, Carthew J, Frith JE, Neild A (2019) Cell adhesion, morphology, and metabolism variation via acoustic exposure within microfluidic cell handling systems. Adv Sci 6(24):1902326

Devendran C, Choi K, Han J, Ai Y, Neild A, Collins DJ (2020) Diffraction-based acoustic manipulation in microchannels enables continuous particle and bacteria focusing. Lab Chip 20(15):2674-2688

Devendran C, Collins DJ, Ai Y, Neild A (2017) Huygens-Fresnel acoustic interference and the development of robust time-averaged patterns from traveling surface acoustic waves. Phys Rev Lett 118(15)

Di Carlo D (2009) Inertial microfluidics. Lab Chip 9(21):3038

Ding X, Lin SCS, Lapsley MI, Li S, Guo X, Chan CY, Chiang IK, Wang L, McCoy JP, Huang TJ (2012) Standing surface acoustic wave (\{SSAW $\})$ based multichannel cell sorting. Lab Chip 12(21):4228-4231

Fakhfouri A, Devendran C, Collins DJ, Ai Y, Neild A (2016) Virtual membrane for filtration of particles using surface acoustic waves (SAW). Lab Chip 16(18):3515-3523

Fakhfouri A, Devendran C, Ahmed A, Soria J, Neild A (2018) The size dependant behaviour of particles driven by a travelling surface acoustic wave (TSAW). Lab Chip 18(24):3926-3938

Fakhfouri A, Devendran C, Albrecht T, Collins DJ, Winkler A, Schmidt H, Neild A (2018) Surface acoustic wave diffraction driven mechanisms in microfluidic systems. Lab Chip 18(15):2214-2224

Faley S, Seale K, Hughey J, Schaffer DK, VanCompernolle S, McKinney B, Baudenbacher F, Unutmaz D, Wikswo JP (2008) Microfluidic platform for real-time signaling analysis of multiple single T cells in parallel. Lab Chip 8(10):1700-1712

Fu YQ, Luo JK, Du XY, Flewitt AJ, Li Y, Markx GH, Walton AJ, Milne WI (2010) Recent developments on $\mathrm{ZnO}$ films for acoustic wave based bio-sensing and microfluidic applications: a review. Sens Actuators B Chem 143(2):606-619

Gascoyne PRC, Vykoukal J (2002) Particle separation by dielectrophoresis. Electrophoresis 23(13):1973

Gor'kov LP (1962) On the forces acting on a small particle in an acoustical field in an ideal fluid. Sov Phys Dokl 6:773-775

Inglis DW, Riehn R, Austin RH, Sturm JC (2004) Continuous microfluidic immunomagnetic cell separation. Appl Phys Lett 85(21):5093-5095

Inglis DW, Davis JA, Austin RH, Sturm JC (2006) Critical particle size for fractionation by deterministic lateral displacement. Lab Chip 6(5):655

Khademhosseini A, Langer R, Borenstein J, Vacanti JP (2006) Microscale technologies for tissue engineering and biology. PNAS 103(8):2480-2487

Kramer SM, Mcbride SL, Mair HD, Hutchins DA (1988) Characteristics of wideband planar ultrasonic transducers using plane and edge wave contributions. IEEE Trans Ultrason Ferroelectr Freq Control 35(2):253-263

Landenberger B, Höfemann H, Wadle S, Rohrbach A (2012) Microfluidic sorting of arbitrary cells with dynamic optical tweezers. Lab Chip 12(17):3177

Lee W, Tseng P, Di Carlo D (2017) Microfluidic cell sorting and separation technology. Springer International Publishing, Cham, pp $1-14$

Ma Z, Collins DJ, Guo J, Ai Y (2016) Mechanical properties based particle separation via traveling surface acoustic wave. Anal Chem 88(23):11844-11851

MacDonald MP, Spalding GC, Dholakia K (2003) Microfluidic sorting in an optical lattice. Nature 426(6965):421-424 
Marzo A, Seah SA, Drinkwater BW, Sahoo DR, Long B, Subramanian $S$ (2015) Holographic acoustic elements for manipulation of levitated objects. Nat Commun

Muller PB, Barnkob R, Jensen MJH, Bruus H (2012) A numerical study of microparticle acoustophoresis driven by acoustic radiation forces and streaming-induced drag forces. Lab Chip 12(22):4617

Nama N, Barnkob R, Mao Z, Kähler CJ, Costanzo F, Huang TJ (2015) Numerical study of acoustophoretic motion of particles in a PDMS microchannel driven by surface acoustic waves. Lab Chip 15(12):2700-2709

Novak R, Ingram M, Marquez S, Das D, Delahanty A, Herland A, Maoz BM, Jeanty SS, Somayaji MR, Burt M, Calamari E, Chalkiadaki A, Cho A, Choe Y, Chou DB, Cronce M, Dauth S, Divic T, Fernandez-Alcon J, Ferrante T, Ferrier J, FitzGerald EA, Fleming R, Jalili-Firoozinezhad S, Grevesse T, Goss JA, Hamkins-Indik T, Henry O, Hinojosa C, Huffstater T, Jang KJ, Kujala V, Leng L, Mannix R, Milton Y, Nawroth J, Nestor BA, Ng CF, O'Connor B, Park TE, Sanchez H, Sliz J, Sontheimer-Phelps A, Swenor B, Thompson G, Touloumes GJ, Tranchemontagne Z, Wen N, Yadid M, Bahinski A, Hamilton GA, Levner D, Levy O, Przekwas A, Prantil-Baun R, Parker KK, Ingber DE (2020) Robotic fluidic coupling and interrogation of multiple vascularized organ chips. Nat Biomed Eng 4(4):407-420

Nyborg WL (1953) Acoustic streaming due to attenuated plane waves. J Acoust Soc Am 25(1):68-75

O'Rorke R, Collins D, Ai Y (2018) A rapid and meshless analytical model of acoustofluidic pressure fields for waveguide design. Biomicrofluidics 12(2):024104

Ozcelik A, Rufo J, Guo F, Gu Y, Li P, Lata J, Huang TJ (2018) Acoustic tweezers for the life sciences

Phan HV, Coskun MB, Sesen M, Pandraud G, Neild A, Alan T (2015) Vibrating membrane with discontinuities for rapid and efficient microfluidic mixing. Lab Chip 15(21):4206-4216

Raymond SJ, Collins DJ, O'Rorke R, Tayebi M, Ai Y, Williams J (2020) A deep learning approach for designed diffraction-based acoustic patterning in microchannels. Sci Rep 10(1):1-12

Regehr KJ, Domenech M, Koepsel JT, Carver KC, Ellison-Zelski SJ, Murphy WL, Schuler LA, Alarid ET, Beebe DJ (2009) Biological implications of polydimethylsiloxane-based microfluidic cell culture. Lab Chip 9(15):2132-2139

Sazan H, Piperno S, Layani M, Magdassi S, Shpaisman H (2019) Directed assembly of nanoparticles into continuous microstructures by standing surface acoustic waves. J Colloid Interface Sci 536:701-709

Sesen M, Alan T, Neild A (2015) Microfluidic plug steering using surface acoustic waves. Lab Chip 15(14):3030-3038

Sesen M, Devendran C, Malikides S, Alan T, Neild A (2017) Surface acoustic wave enabled pipette on a chip. Lab Chip 17(3)

Settnes M, Bruus H (2012) Forces acting on a small particle in an acoustical field in a viscous fluid. Phys Rev E Stat Nonlinear Soft Matter Phys 85(1)

Shafiee H, Sano MB, Henslee EA, Caldwell JL, Davalos RV (2010) Selective isolation of live/dead cells using contactless dielectrophoresis (cDEP). Lab Chip 10(4):438

Tayebi M, O'Rorke R, Wong HC, Low HY, Han J, Collins DJ, Ai Y (2020) Massively multiplexed submicron particle patterning in acoustically driven oscillating nanocavities. Small 16(17):2000462

Tsou JK, Liu J, Barakat AI, Insana MF (2008) Role of ultrasonic shear rate estimation errors in assessing inflammatory response and vascular risk. Ultrasound Med Biol 34(6):963-972

Vanneste J, Bühler O (2011) Streaming by leaky surface acoustic waves. Proc R Soc A Math Phys Eng Sci 467:1779-1800

Wang Y, Zhao Y, Cho SK (2007) Efficient in-droplet separation of magnetic particles for digital microfluidics. J Micromech Microeng 17(10):2148-2156

Xia N, Hunt TP, Mayers BT, Alsberg E, Whitesides GM, Westervelt RM, Ingber DE (2006) Combined microfluidic-micromagnetic separation of living cells in continuous flow. Biomed Microdevice 8(4):299-308

Xu H, Dong S, Xuan W, Farooq U, Huang S, Li M, Wu T, Jin H, Wang X, Luo J (2018) Flexible surface acoustic wave strain sensor based on single crystalline LiNbO3 thin film. Appl Phys Lett 112(9):093502

Zervantonakis IK, Kothapalli CR, Chung S, Sudo R, Kamm RD (2011) Microfluidic devices for studying heterotypic cell-cell interactions and tissue specimen cultures under controlled microenvironments. Biomicrofluidics 5(1):13406

Zhang Y, Devendran C, Lupton C, de Marco A, Neild A (2019) Versatile platform for performing protocols on a chip utilizing surface acoustic wave (SAW) driven mixing. Lab Chip 19(2):262-271

Publisher's Note Springer Nature remains neutral with regard to jurisdictional claims in published maps and institutional affiliations. 